\title{
Limits of Space Syntax for Urban Design: Axiality, scale and sinuosity
}

\author{
Abstract \\ Space syntax analysis of the city as a movement economy has made major contributions to \\ our understanding of the spatial structure of cities, particularly the importance of a mapping \\ of network integration in relation to density, functional mix and streetlife vitality. It has \\ focused attention of urban researchers onto the importance of the relations between the \\ sociality and spatiality of the city. The primary methods of syntactic analysis involve a \\ reduction of urban morphology to a set of spatial axes; here we explore some limits to such \\ analysis for urban design. Topological analysis of axial models has long recognized problems \\ in accounting for distance, scale and sinuous streetscapes. Existing adaptations to axial \\ methods that address such problems are modelled and shown to produce a broad range of \\ results for the same urban morphology. In each case we also compare different capacities for \\ to-movement and through-movement - the distinction between 'closeness centrality' and \\ 'betweenness centrality' that shows that network integration is multiple. We argue that axial \\ analyses privilege visibility over accessibility and can produce distorted mapping at walkable \\ scales; only one of the methods tested measures permeability and walkable access. Space \\ syntax analysis is a powerful tool that will be more useful the better such limits are \\ understood.
}

\section{Keywords}

Space syntax, urban morphology, urban design, multi-scale, street network 


\section{Introduction}

Space syntax analysis as developed by Hillier (1996a) and colleagues has become the most influential method of analysing street networks as spaces of pedestrian movement (Hillier and Hanson, 1984; Hillier, 1996b; Hillier and Vaughan, 2007). Space syntax involves a focus on the configurational aspects of spaces in buildings and towns - on the arrangement or assemblage of urban elements into networks of connectivity. For Hillier the 'movement economy' is fundamental to the ways that cities work, and "movement in the urban grid is, other things being equal, generated by the configuration of the grid" (Hillier, 1996a: 5). The 'topological' or 'syntactic' properties of this model are then measured using specifically developed software and represented through colour maps. A key measure of network properties is the degree to which each part of a network is spatially integrated with the larger network. Integration is thus a measure of urban connectivity and accessibility that can be measured by distance, by the number of nodes one must pass through, or the number of axial links.

The usefulness of integration measures is twofold. First, locations with high levels of network access generally attract higher flows of movement, higher density development and higher levels of land-use mix. This nexus of connectivity, concentration and co-functioning tends to produce high levels of streetlife and 'co-presence' in public space. Locations with a high level of integration are where the public life and sociality of the city is most intensive, they are more 'urbanized'. Thus it is as a general theory of urbanity that space syntax offers the most promise.

\footnotetext{
"Well-functioning cities can... be thought of as 'movement economies'. By this is meant that it is the reciprocal effects of space and movement on each other... and the multiplier effects on both that arise from patterns of land use and building densities, which are themselves influenced by the space-movement relation, that give cities their characteristic structures, and give rise to the sense that everything is working together to create the special kinds of well-being and excitement that we associate with cities at their best." (Hillier, 1996a: 153)
} 
While we acknowledge the contributions that space syntax analysis has made to our understanding of cities, we seek to explore the limits of such an approach both theoretically and methodologically. We are concerned about the degree of reductionism and determinism we see as embodied in the space syntax literature, the premise that urbanity can be explained by one basic universal principle, that "physics is the theory of everything" (Nagel, 1998). We argue that social phenomena can emerge at any spatial scale (DeLanda, 2006), and the mezo-scale of the walkable neighbourhood is one of them. We will also argue that the methods of analysis can be blunt, inaccurate and misleading; and that they do not cope well with complexities of scale, topography, 3-dimensionality and multi-modal movement. We are also alarmed at the degree to which students apply space syntax mapping as if it were an end rather than a means to urban analysis. As such practices are often a result of poor understanding of the theory, here we aim to avoid unnecessary jargon.

Space syntax analysis is not a singular method but a cluster of approaches to the analysis of spatial relations - mainly gamma, isovist and axial analyses. While our focus here is on the last of these, with its variations of segment and road centre-line analysis, in order to place this critique in context we begin with a sketch of all three approaches. 'Gamma analysis' primarily involves a diagrammatic mapping of relations between spatial segments of building interiors, with rooms and spaces conceived as cells with the building entry as a base (Hillier and Hanson, 1984: 142ff) (Figure 1a). This work has become seminal in our understanding of the ways that space constructs social meaning in architecture - particularly the understanding of power relations, the overall depth of buildings and the relative depth or shallowness of particular cells within. Gamma analysis can be used to calculate the degree of integration of each 'cell' - the level of connectivity with all other cells within that building. Gamma analysis often involves a quest to identify the morphogenic patterns or genotypes of spatial structure that underlie the production of space in common building types. Depth and shallowness of spatial structure can be revealing of how buildings mediate relations between insiders and outsiders. A key insight lies in the difference between public space which is primarily 'ringy', networked and designed to promote flows of strangers, and interior space which is generally more tree-like with much stronger forms of ideological control, designed to mediate asymmetric relations of power - particularly between insiders and visitors (Hillier and Hanson, 1984: 102). Markus (1993) shows how this works in the disciplinary technologies of prisons, 
workhouses, schools, hospitals and asylums as well as museums and theatres of the enlightenment. Gamma analysis is primarily applied to interior space, based in large part on the assumption that interior space is cellular: "by and large, a building consists of welldefined spaces with well-defined links from one to another" (Hillier and Hanson, 1984: 16). Yet for public space that is rarely the case.

(A.)
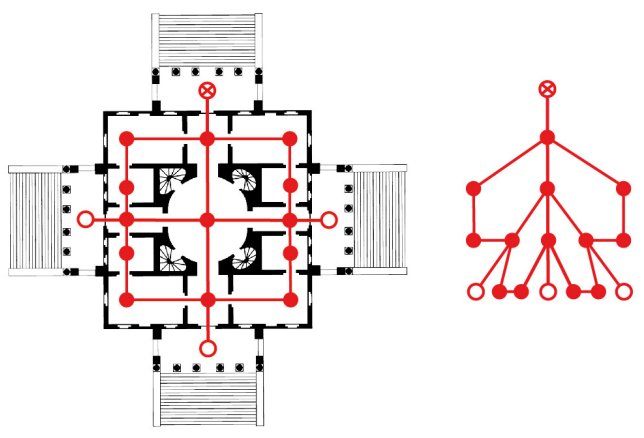

(B.)

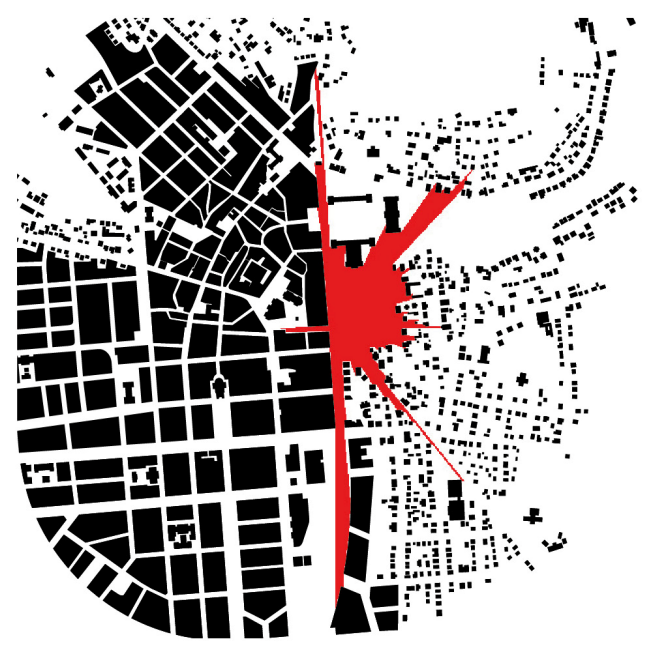

(C.)

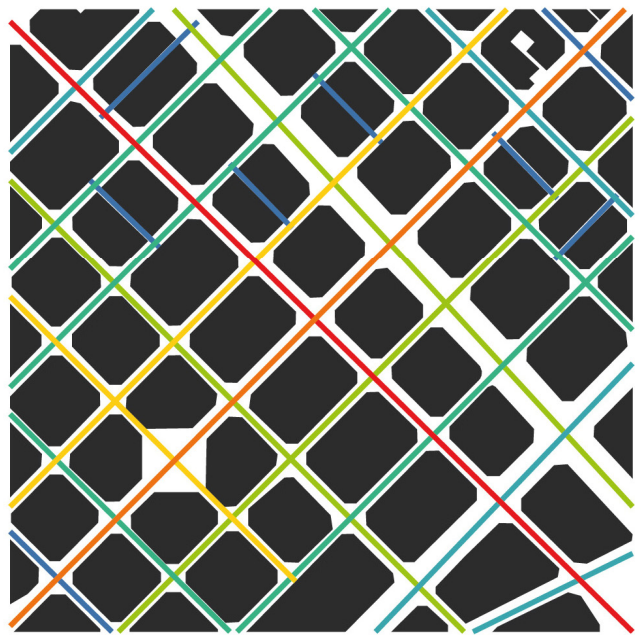

Figure 1. Three approaches: (a) gamma, (b) isovist and (c) axial analysis. 
Isovist mapping is an analysis of the area of space that can be viewed horizontally from any single location, generally understood to be the field of view that is not blocked by walls or buildings. Thus an isovist relates to a visual phenomenology of space - it determines the extent of the visual field that is enabled or constrained by architecture or urban design (Figure 1b). In practice that visual field is also mediated by vehicles, trees, street signage and furniture at short range and by topography, light and air quality at longer range. Isovist mapping reveals those locations with the largest fields of view, the ways architecture mediates privacy and the ways the gaze of the visitor is constructed. The isovist map measures the degree to which the larger spatial structure can be seen from a single location. However, it is not a measure of access since isovists extend through transparent borders that cannot be crossed.

While gamma analysis involves a focus on topological relations between cells, and isovist analysis involves fields of visibility, axial analysis extends this idea of lines of sight to calculate the minimum number of straight axes necessary to navigate the network of public spaces. Each axis is considered as one element of the spatial network regardless of length and is allocated a single integration value. The most integrated axes are those with the least number of axial turns needed to connect to all other axes in the network. The relative value for each street within the system is represented in a colour spectrum from red (high integration) through orange, yellow, and green to cyan and blue (low integration) (Figure 1c). Variations of this method include analysis of segment models, where axial lines are divided at each intersection, and road centre-line models, based on the actual geometric axis of the street (Al-Sayed et al., 2014).

These three approaches to space syntax differ both in methodology and scale of application. Gamma analysis is largely limited to architectural scales, isovist analysis extends also to urban design scales while axial analysis can be used across all scales. With variations and refinements, axial analysis has become the most commonly applied method of space syntax analysis for urban space and is the primary subject of our critique. The arguments that follow are multiple and often interconnected; we will proceed through a series of questions. To what degree can urbanity be reduced to, or determined by, the spatial network? How effectively can a topological method incorporate questions of distance? To what degree does 
space syntax analysis produce useful results at walkable neighbourhood scale? How do axial methods deal with sinuous streets? These last three questions are explored through mapping of particular urban morphologies. We conclude with a discussion of the limits revealed here and of further challenges in dealing with three-dimensional topographies, micro-morphologies and multi-modal access networks.

\section{Reductionism and Urbanity}

Netto (2016) has argued that there are three significant forms of reductionism embodied in the space syntax approach: the reduction of spatial practices to movement; the reduction of social interaction to bodily presence in public space; and the reduction of urban space to its syntactic properties. These might be collectively summarized as the reduction of urbanity to the spatial network. Hillier's theory of urbanity has most succinctly been put as follows:

'Urbanity... is not so mysterious. Good space is used space. Most urban space use is movement. Most movement is through movement, that is, the by-product of how the grid offers routes from everywhere to everywhere else. Most informal space use is also movement related, as is the sense and the fact of urban safety. Land uses and building density follow movement in the grid, both adapting to and multiplying its effects. The urban buzz, or the lack of it when it suits us, is the combination of these, and the fundamental determinant is the structure of the grid itself." (Hillier, 1996a: 170)

This is an insightful account of how cities work that integrates a great deal of good urban theory; the key importance attributed to movement networks is neither new nor contested here. In the 19th century Cerdá's 'general theory' of urbanity was based in a synergy between streets, buildings and people, reflected in the neologism 'viality' - a property that combined street (via) with vitality (Soria y Puig, 1999: 103-115). Streetlife vitality is one property of all good public space and Hillier's work shows that the movement economy is strongly geared to spatial structure. While most streetlife is comprised by movement, most other activities in public space also depend upon traffic flows to a significant degree. Urban safety is better managed by co-presence than by walls - an insight embodied in 'The Social Logic of Space' (Hillier and Hanson, 1984) that extends Jacobs' (1961) notion of 'eyes on the street'. 
Functional mix and building density are generally attracted to the more integrated parts of the network where they also become urban attractors in turn. We share the desire for a better understanding of the qualities of urbanity and urban buzz that are at once so clearly valued and productive yet elusive. We baulk, however, at the idea that urbanity might be reduced to any 'root' cause - the assertion of spatial structure as the 'fundamental determinant' of what makes a city tick. This primary role attributed to street network configurations ahead of other morphological dimensions and social practices is the aspect of space syntax that has been most widely contested (Holyoak, 1996; Netto, 2016; Ratti, 2004; Soja, 2001).

\section{Distance and Scale}

Axial analysis, as initially developed by Hillier and Hanson (1984), is a topological approach that largely ignores distance to focus on relations of interconnectivity. The lines of movement (streets as axes) become the primary elements (or nodes) of the network with the intersections as links. This enables such analysis to account for the continuity of urban streets that is crucial for understanding movement economies, yet when operationalized as analysis the spatial network is reduced to axial lines of sight. As Marshall (2005: 115) puts it: "This appeal to connectivity and continuity seems to point essentially to the importance of lines of movement - but does not seem inevitably to point to 'axial lines of sight'..." In other words the spatial axis is a proxy that can be used to reduce highly differentiated urban morphologies to numeric calculation. This reduction of lines of movement to lines of sight is at the heart of several limits to space syntax analysis.

As a topological measure axial analysis discounts physical/spatial distance, and generates a single integration value along the entire length of a long axis. This has been justified on the basis that the desire to move in straight lines prevails over choosing the shortest distance. Hillier and Vaughan (2007: 216) argue that people do not navigate in accordance with metric distance but according to the paths with the 'least angle' of change. They have evidence that this is the case in London but such an argument surely has limits. Such tendencies are more likely to be relevant in areas where most pedestrians are visitors, and thus visual connectivity (legibility) is more relevant. Local residents with a well-formed mental map will probably choose the shorter route over the globally integrated one (Ratti, 2004). As Steadman (2004: 
484) points out: "many published space syntax maps show axial lines for streets which do not in reality afford a view from one end to the other because they pass over hills, or extend over many kilometres". There is a further problem in that the simple axial method suggests that we are equally integrated at any position along the axis. The long axes of any city will pass through a range of different urban morphologies - large- and small-grain, mixed and monofunctional, more- and less-dense, with differences of traffic volume.

Since any calculation of integration is relative to the whole network the question of where one draws a boundary around the network is crucial. This is the problem most succinctly put by Ratti (2004) who demonstrates that two neighbourhoods or towns with a single interconnection will produce different integration levels depending on whether they are analysed as two networks or one. Only in small towns, gated neighbourhoods or islands is there a clear edge to the network and fundamentally different integration measures emerge depending on where one draws the boundary of the network. Spatial syntax analysis can be very insightful under conditions where an access network is relatively clearly bounded such as a whole city or a building or enclave within it; yet it can also be problematic at neighbourhood scale.

The problems produced by distance and scale have long been recognized within spatial syntax research and there have been several adaptations to address them. One response has been to develop measures of 'segment integration' whereby each axis is divided into segments that terminate at each intersection enabling differential measures of integration along extensive straight axes (Dalton et al., 2003). This method, however, will also produce differential outcomes according to how 'streets' and 'intersections' are defined - when does a laneway become a street? An axis adjacent to a long block is equated to that adjacent to a short block, so distance is again discounted. A second approach in this regard has been to combine topologic and metric analysis to produce 'local' integration measures within a restricted 'integration radius' - a 'radius' that may be measured as a number of axial steps, the total distance along the axes, or the total amount of angular change. Thus, while the network that is mapped will be larger than this radius, the calculation for each axis is local. Hillier et al. (2007) have argued that configurational properties of urban networks are dualistic: metric at 'local' scale and topo-geometric at 'global' scale. Axial integration 
properties are increasingly combined with metric properties, by limiting the integration radius to a given metric value (Al-Sayed et al., 2014). This begs the question of what such a limit might be - walking distances are elastic and it is difficult to establish any such limit that is not arbitrary. Approaches such as these help to address both the distance and boundary problems. A further adaptation to such methods known as angular segment analysis will be discussed in a later section.

\section{Closeness and Betweenness}

These methods of measuring integration are complicated further by the distinction between 'to-movement' and 'through-movement', a distinction between streets that are central to other streets in the network (to-movement or integration) and the streets that are chosen most as a shortest path between other streets in the network (through-movement or 'choice') (Hillier and Vaughan, 2007: 213-214). This is a distinction that predates space syntax theory, known in network theory as closeness centrality and betweenness centrality. Thus these are each measures of network centrality, yet within space syntax discourse the former is called integration while the latter is termed 'choice'. Integration analysis measures the least number of axes or segments encountered in moving from any single axis to all of the others in the network; choice analysis measures the degree to which each axis lies on the simplest path between all possible pairs of axes (all possible trips) within that network (Hillier and Vaughan, 2007: 214). If one were looking for a location to maximize access to all other parts of the network then one would analyse for closeness (to-movement or integration); on the other hand if one were to choose on the basis of locating adjacent to through traffic one would analyse for betweenness (through-movement or choice). We find this nomenclature confusing because 'integration' is narrowly defined as one kind of 'centrality' when 'betweenness' is surely another kind of integration. Confusion has been created in the literature by contrasting 'integration' with 'choice' when in Hillier's (1996a: 170) words "most movement is through movement". This is compounded by the fact that the axes with the highest through movement (such as bridges and freeways) are those where we get little choice. For clarity we will use the original terms: closeness centrality (for to-movement or integration) and betweenness centrality (for through-movement or choice). 
While there are further complexities we might seek to unravel here, this is enough to test the limits of space syntax on some particular urban morphologies. To summarize the complexities thus far: not only are there three primary methodological approaches (gamma, isovist and axial), but axial methods can be adjusted in several different ways (segmentation and radius), and each of these can measure closeness centrality or betweenness centrality .

\section{Walkable Access}

We now turn to the question of how well space syntax analysis works for understanding walkable access at neighbourhood scale. Here we want to test the way a single morphology with heterogeneous block size can be mapped using different measures. We are not suggesting that walkability can be reduced to block size, only that it can be a useful proxy for testing these measures. Figure 2 applies six different methods of analysis to a single abstract morphology that is designed with two grids of $1 \times 1 \mathrm{~km}$ each and linked by two bridges. The number of axial segments in each grid is identical, but there are more axial lines in the righthand grid (with many dead-end streets), and thus permeability is lower (the average block perimeter is double). 'Axial closeness' (integration) and 'axial betweenness' (choice) analysis (Figures $2 \mathrm{a}$ and $2 \mathrm{~b}$ ) produce higher results on the right because the number of axial lines is larger; this shows that neither of these are measures of permeability. The second row of diagrams (Figures 2c and 2d) analyse 'segment closeness' and 'segment betweenness' without applying a radius limit. They show a similar level of integration for both grids, and a high level of both closeness and betweenness for the 'bridges', where permeability is low. The final row (Figures $2 e$ and $2 f$ ) applies a segment radius of $400 \mathrm{~m}$ (a roughly walkable distance) to account for scale. The map of 'segment closeness' (Figure 2e) shows values peaking at the centre of each grid but more strongly in the more permeable left-hand grid, with the bridges having no major impact. In contrast the map of 'segment betweenness' (Figure $2 f$ ) shows the highest values at the bridges and in the less permeable grid. The methods used to produce these maps are identical to those routinely used by space syntax practitioners. 


\section{(A.) AXIAL CLOSENESS}

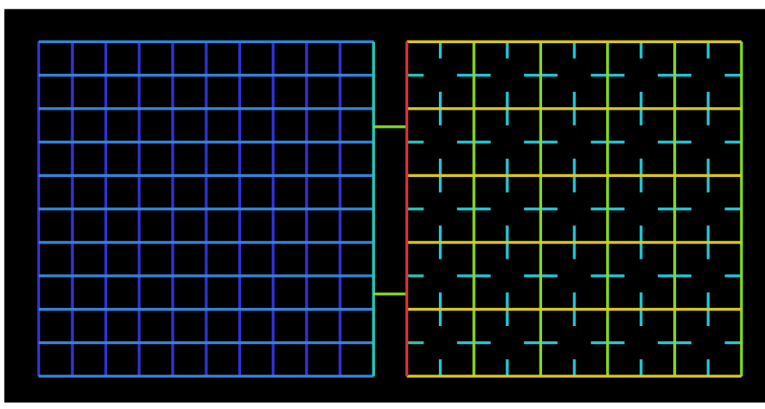

(C) AXIAL SEGMENT CLOSENESS

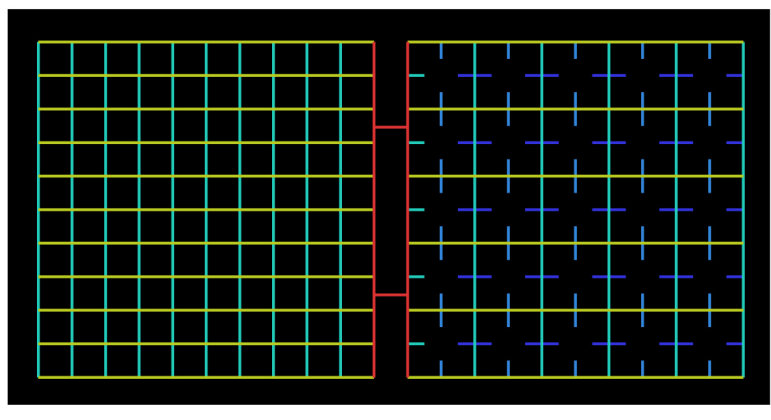

(E) AXIAL SEGMENT CLOSENESS RADIUS $400 \mathrm{M}$

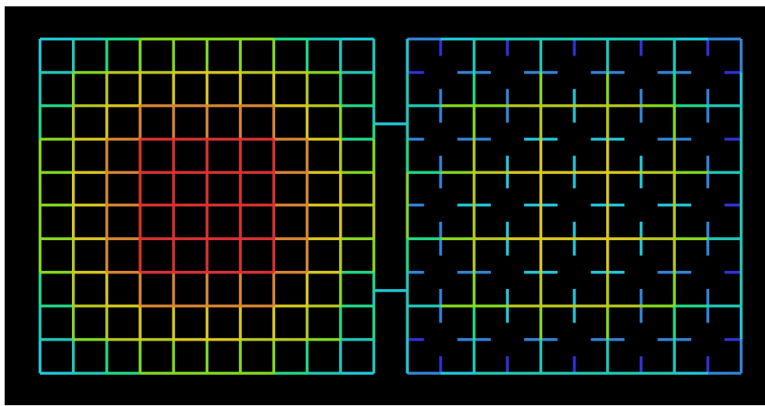

HIGH LOW
(B) AXIAL BETWEENNESS

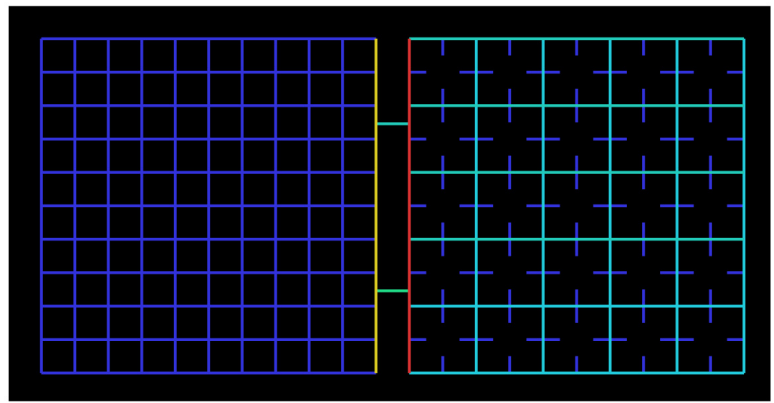

(D) AXIAL SEGMENT BETWEENNESS



(F) AXIAL SEGMENT BETWEENNESS RADIUS $400 \mathrm{M}$

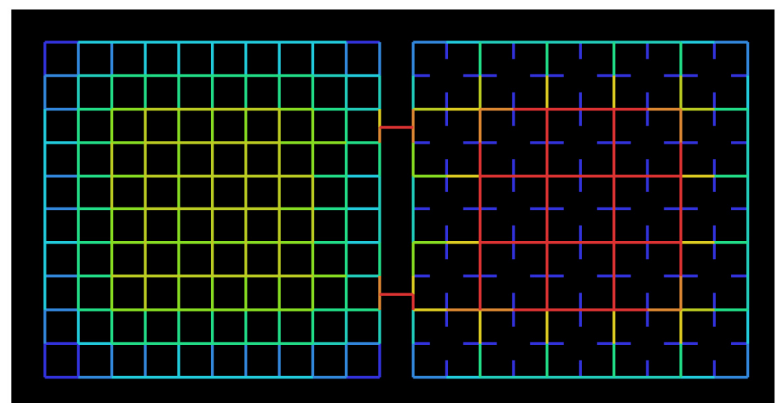

Figure 2. One morphology/six measures of centrality (integration).

These diagrams demonstrate that only one of the six measures (2e) approximates a mapping of permeability that analyses block-size alone (a single peak in the centre of the left hand grid). These results are strongly influenced by the cul-de-sacs and the segregation of the two grids; if both were taken out the results would be more convergent with block size. However we designed the model to reflect the diversity of urban conditions we find in real cities with freeways, escarpments and waterways. We conclude that these methods only measure walkable access when limited to closeness centrality and after segmentation and a walkable radius are applied. 
We now want to test some of the effects of scale on axial mapping and in this case we use a real urban morphology. Figure 3 shows segment analysis of closeness centrality for the vehicular network of central Melbourne where each of three $1 \times 1 \mathrm{~km}$ maps draws data from networks of increasing scale: from $1 \times 1 \mathrm{~km}$, to $2 \times 2 \mathrm{~km}$ and $5 \times 5 \mathrm{~km}$. It demonstrates the ways in which segment closeness (integration) measures change with expanding and contracting scales. While there are some consistencies of measures across different scales there are also streets that change dramatically from poorly integrated at one scale to highly integrated at another and vice-versa. On the one hand this can be seen as a problem for space syntax since any claim that the qualities of urbanity - the density, buzz and mix - are determined by integration clearly begs the question of integration at what scale? On the other hand it demonstrates the value of such methods in revealing the differential role of any street segment at different scales. A street may be highly integrated at neighbourhood scale $(1 \times 1 \mathrm{~km})$ and much less so for car transport. 

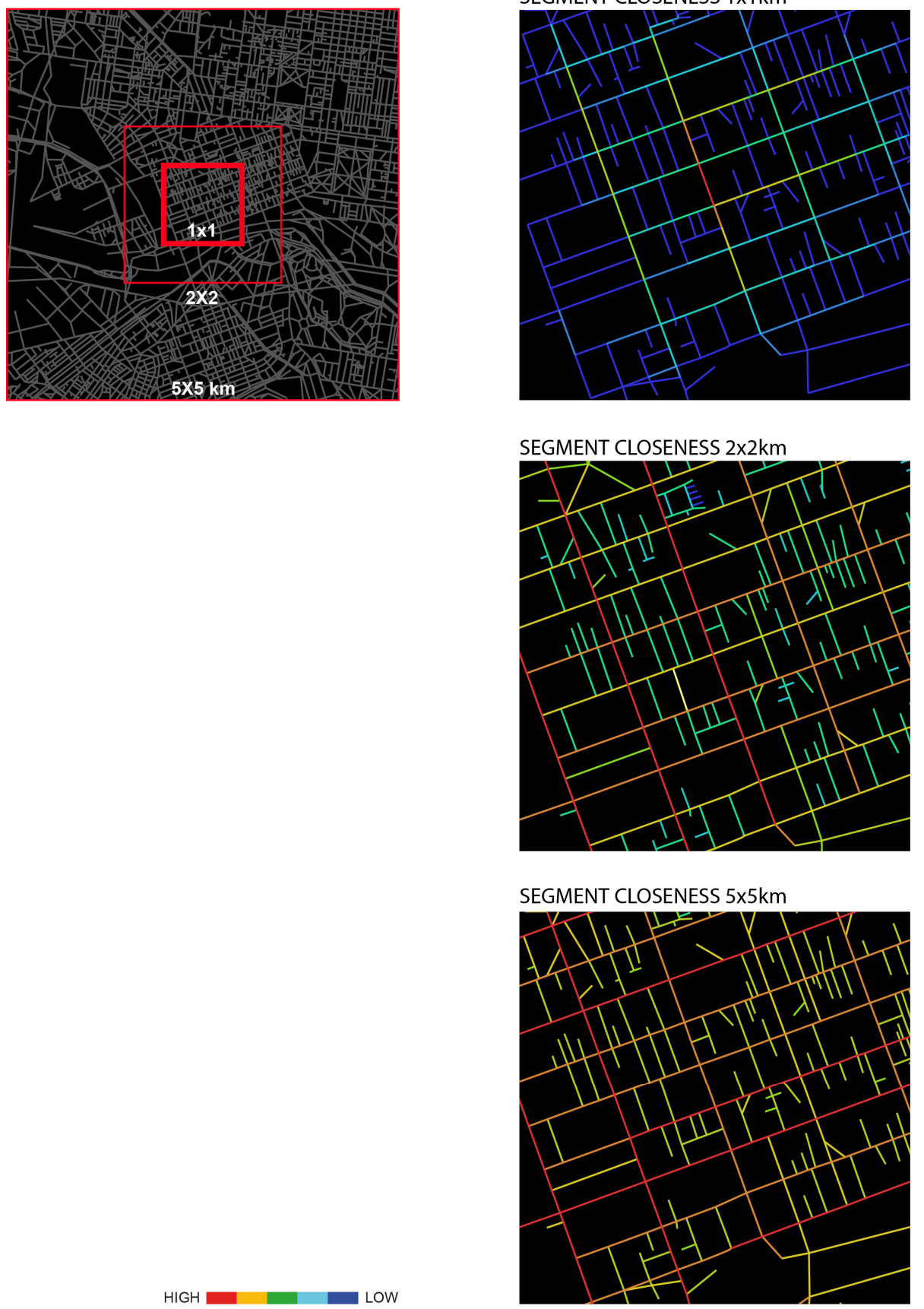

Figure 3. Melbourne closeness centrality (integration) at three scales.

\section{Sinuosity}

We now turn to a further problem with axial analysis that might be called the problem of sinuosity. A key limitation of the axial space syntax approach is revealed by the various attempts to model curved streets such as Broadway in Manhattan that meanders across the grid producing quite different integration values than the straight streets with which it intersects. The reduction of curved streets to a minimum series of axial lines leads to much lower integration values than a single straight street would have, even when the experienced 
sense of continuity is profound - as in Broadway. We detect five main approaches to the problem of sinuosity. First, it can be dealt with under 'segment integration' as outlined above - the sinuous street becomes segmented by intersections. Second, changes in direction below a threshold angle can be considered as insignificant (Figueiredo and Amorim, 2005). A street like Broadway would become a single axial line, yet the threshold for discounting an angular turn is arbitrary while visual continuity is gradual and dependent on street width. A third response involves an 'angular segment analysis' that differentiates between various degrees of turns and aggregates them (Al-Sayed et al., 2009). In this approach three $30^{\circ}$ turns equal one $90^{\circ}$ turn. Here Broadway would be treated as near straight but the problem of metric distance emerges again - a street that curves through $90^{\circ}$ over $20 \mathrm{~m}$ will register the same as one that turns over $200 \mathrm{~m}$. This has been addressed by weighing each angular turn by the length of its segment (Turner, 2007). Broadway is an example that illustrates both the problems of simple axial analysis and the advantages of angular segment analysis.

Another approach to the problem of sinuosity is labelled 'intersection continuity negotiation' (Porta et al., 2006). Here the basic elements of the network are defined as the most easily negotiated paths, considering that at each intersection the straightest direction (least angle) is chosen. The result will depend on an arbitrary decision about where the computation of paths begins. Finally, there is an approach that we might call 'named-street analysis' where a street with a continuous name is represented as an axis regardless of the configuration (Jiang and Claramunt, 2004). Here the problem of defining continuity leaps from morphological analysis to discourse analysis but it is a somewhat simplistic approach that serves to exemplify the difficulty of reducing real cities to topologic models. To compound the complexity, of course each of these approaches can be used to map closeness centrality (tomovement) or betweenness centrality (through-movement).

Figure 4 demonstrates the ways that the 'angular segment' approach can be mapped on the relatively sinuous morphology of Amsterdam at a scale of $1 \times 1 \mathrm{~km}$ (Figure $4 a)$. It shows how 'angular segment' analysis can be mapped for both closeness centrality (Figure 4c) and betweenness centrality (Figure 4d) as compared with the simple axial method (Figure 4b). The simple axial map shows a main spine along the Dam as most integrated. When the angular segment method is applied for closeness centrality it then shows high integration 
values extending across the canal zone with the highest values for streets that align with the bridges. The betweenness map is similar but with a far greater contrast between streets aligned with canals and those aligned with bridges. None of these patterns reflect the contrast between the small blocks of the central city (on the right) and the large blocks of the canal zone (on the left), reinforcing again that such methods are not mapping permeability or walkability.

\section{A. STREETSPACE}



C. ANGULAR SEGMENT CLOSENESS



B. AXIAL CLOSENESS

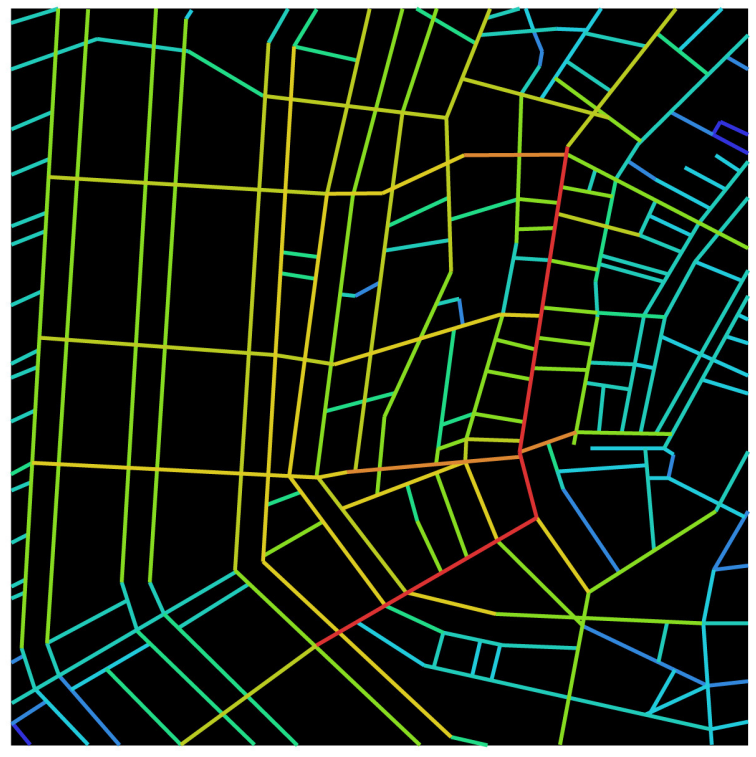

D. ANGULAR SEGMENT BETWEENNESS

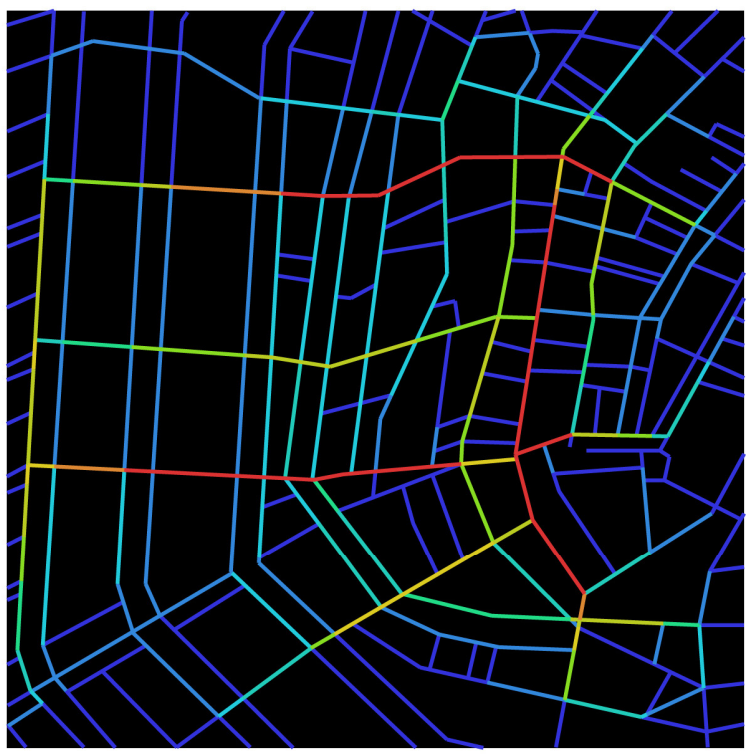

HIGH L LOW

Figure 4. Capturing sinuosity - Amsterdam. 


\section{Discussion}

So what have we achieved in this paper? We have shown that axial methods of space syntax analysis produce a broad range of results depending on which method is used, the scales at which they are applied and variegations of the morphology. We have demonstrated that axial methods can produce problematic results at walkable scales and do not measure permeability. The surgical end of our analysis can be found in the maps and our critiques of them. We hope to have brought some clarity to the understanding of space syntax methods for the broader fields of urban research and of the limits of current practice.

The axial methods we have explored include the original axial analysis, angular segment analysis and angular segment analysis with a radius, each applied to measures of 'integration' (to-movement) and 'choice' (through-movement). These properties of 'integration' and 'choice' are essentially synonyms of 'closeness centrality' and 'betweenness centrality' in network theory and they are confusingly renamed within the field of space syntax. 'Choice' has the highest values for those streets that we have no choice but to use (Figure 2). The use of the term 'integration' is also confusing since closeness and betweenness are different kinds of 'centrality', different ways in which links in the urban spatial structure are more or less integrated.

The distinction between closeness and betweenness becomes crucial when we consider the relations between space syntax and permeability. Defined as the capacity for flows to permeate an urban fabric, permeability has long been a mainstream criterion for urban design practice and is often used as a synonym for connectivity in a more general sense. Jacobs (1961) introduced the principle of 'short blocks' and while various metrics have developed since then (Pafka and Dovey, 2017), space syntax is not one of them. Permeability is a property embodied in a patch of the urban network at whatever scale; it is not a property of a particular street or link within that network. Unlike measures of network centrality, permeability is a property that is largely readable on a map. A regular continuous grid will always show equal permeability across the grid while measures of centrality always peak at the centre. Only one of the axial methods we tested - the 'angular segment' method of mapping 'closeness' with a walkable metric radius - can produce results similar to permeability measures (Figure 2e). It follows that permeability is more geared to closeness 
than to betweenness; that an understanding of betweenness is more useful at larger scales and can be misleading at walkable scale. While there is often congruence between centrality and permeability, and axial analysis can usefully model how changes to permeability will impact on centrality, the two should not be confused.

Any mapping of the city must reduce the complexities of urban form to particular layers of data - the power of the method and the map can derive from this choice of data as do its limits (Dovey et al., 2018). In this case the primary data are spatial axes. Turner (2007) has argued that such reduction can go further, that the use of road-centre lines instead of axial lines can produce a better correlation with vehicular movement. As always, however, the effectiveness of the method depends on the research questions. If we are to better understand walkable networks then the particularities of urban morphology become more important and the commonly available databases reach their limits. The micro-morphology of the street, including sidewalks, tree planting, fences, traffic and so on are necessarily ignored when abstracting the street section to its axis. Micro-scale attractors and obstacles to pedestrian flow, as well as connectivity between the two sides of the street are ignored. It is well established that sociality in public space relates to micro-morphological elements (Jacobs, 1961; Whyte, 1980) and that the social logic of the street is not just longitudinal but also transversal across the street (Appleyard, 1981; Cerdá, 1867). Cities are often threedimensional networks of overpasses and underpasses that can be very difficult to reduce to spatial axes. Urban connectivity is also a multi-modal mix of walking, cycling, driving and public transport using a mix of shared and dedicated networks. While there are incipient attempts to integrate dedicated public transit networks into space syntax analysis (Law et al., 2012; Goodship, 2015; Gil, 2014), there is unlikely to be a single method of spatial analysis that can effectively deal with multi-modality, three-dimensionality and micro-morphology.

We have also shown how axial analysis produces maps that differ significantly with the scale of analysis - the main street that commands the highest centrality/integration values at one scale can become part of a segregated neighbourhood when the scale expands. These effects of scale then intersect in complex ways with measures of closeness and betweenness. While this will not surprise space syntax researchers, it shows again that integration/centrality are plural properties. 
Many of these limits are understood by space syntax practitioners, particularly those of distance, scale and sinuosity that have plagued the earlier axial approaches. However, they can be misunderstood from outside that field and the various methodological adaptations invented to deal with them have also produced a significant level of opacity. One needs to learn the language and the methods in order to engage effectively, yet once one does so the audience is narrow indeed. Terms of expression often slip between mathematical formulae, graph theory and space syntax jargon. Jargon in any field can be necessary but it is also one of the micro-practices of power that turns ideas into ideologies and defends them from attack. One result is that space syntax analysis is often used by those with no more understanding than of how to match the morphological data with the software. Axial maps are presented as the answer to how the city works in terms of spatial structure - a 'science' of urban design that is largely accepted on faith by those with little understanding of the methods, issues of scale, multi-modality or even database accuracy. A part of our goal here has been to bring some clarity in this regard but we cannot claim success because these issues get more complex the deeper one explores them.

The title of Hillier's (1996a) book 'Space is the Machine' is a play on Corbusier's famous proclamation of architecture as a 'machine for living'. Corbusier was in thrall to the sleek machine aesthetic of yachts and aircraft where design emerges from finely-tuned relations with the material forces of nature and the desire for speed. Yet his modernist aesthetic was never formed from a fine-tuned relation with function since the functions of architecture cannot be reduced in this way. This is also true for cities and raises the question of the degree to which the city can be reduced to a science (Dovey and Pafka, 2016). For Hillier space is the machine that produces urban intensity and his quest is to produce a science of cities from the study of 'natural movement'. In this regard we would like to go part way with Hillier - space is 'machinic' in that it has multiple properties that are geared to each other in productive alliances (Deleuze and Guattari, 1987: 17). However, any claim that spatial configuration is the engine that drives the larger assemblage is much more difficult to support. 
One of the key contributions of space syntax analysis lies in demonstrating that axial maps often correlate with the distribution of a range of activities within the district, levels of pedestrian flow, density and land use mix (Hillier and Vaughan, 2007). Within this assemblage of density, mix and access networks, cause-effect relations can be difficult to discern. While densities of all kinds - buildings, populations and streetlife - will be attracted to more integrated locations, it is also the case that access networks are designed and adapted to connect attractive locations and areas of high density. Arcades emerge within cities to create shortcuts between existing attractions. Likewise functional mix or co-functioning is a set of relationships that may be imposed by zoning or may co-evolve with the access network. Streets, freeways and public transport are often designed in order to connect disparate attractions just as those functions are attracted to the more integrated streets. Density, mix and access networks are co-dependent, they co-evolve as part of an urban assemblage (Dovey and Pafka, 2014; 2017) that cannot be reduced to the spatial network as the 'fundamental determinant' (Hillier, 1996a: 170).

Space syntax is a powerful analytic tool that we would hope to see developed in more sophisticated ways and with a stronger multi-disciplinary engagement with the social concerns from which it originally emerged - maybe a little less reduction of the complexities of urban space to numbers. It is the maps rather than the numeric integration scores that are most revealing in the space syntax approach. Hillier and Vaughan's (2007) review of the development of space syntax is entitled 'The City as One Thing', by which they indicate an integration of "the physical city and the social city". Space syntax was invented to bridge this divide - a social logic of space - to investigate and understand the ways in which spatial configuration is inherently social. Hillier's work in general is driven by the desire to correct what he sees as the tendency of contemporary social theory to point out the social importance of urban space but then to discount the importance of space 'in itself' (Hillier, 2008). This approach has been seminal in our understanding of the ways in which urban morphology is geared to social integration, how segregation is linked to practices of power, and to better understand how cities work. Our argument is not that space syntax is a wrong way to map the city, only that there are multiple ways of mapping cities and spatial structures - not one right way - and they all have their limits. 


\section{References}

Al-Sayed K, Turner A and Hanna S. (2009) Cities as Emergent Models: The Morphological Logic of Manhattan and Barcelona. In: Koch D, Marcus L and Steen J (eds) 7th International Space Syntax Symposium. Stockholm: KTH.

Al-Sayed K, Turner A, Hillier B, et al. (2014) Space Syntax Method. London: Bartlett School of Architecture, UCL.

Appleyard D. (1981) Livable Streets. Berkeley: University of California Press.

Cerdá I. (1867) Teoria General de la Urbanizacion. Madrid: Imprenta Española.

Dalton NS, Peponis J and Dalton R. (2003) To tame a TIGER one has to know its nature: extending weighted angular integration analysis to the description of GIS roadcenterline data for large scale urban analysis. 4th International Space Syntax Symposium London, 65.1-65.10.

DeLanda M. (2006) A new philosophy of society. London: Continuum.

Deleuze G and Guattari F. (1987) A Thousand Plateaus. London: Athlone.

Dovey K and Pafka E. (2014) The urban density assemblage: Modelling multiple measures. Urban Design International 19(1): 66-76.

Dovey K and Pafka E. (2016) The science of urban design? Urban Design International 21(1): 1-10.

Dovey K and Pafka E. (2017) What is functional mix? Planning Theory \& Practice 18(2): 249267.

Dovey K, Pafka E and Ristic M. (2018) Mapping Urbanities: Morphologies, Flows, Possibilities. New York: Routledge.

Figueiredo L and Amorim L. (2005) Continuity lines in the axial system. In: Nes Av (ed) 5th International Space Syntax Symposium. Delft: Techne Press.

Gil J. (2014) Analyzing the Configuration of Multimodal Urban Networks. Geographical Analysis 46: 368-391.

Goodship P. (2015) The impact of an urban cable-car transport system on the spatial configuration of an informal settlement. The Case of Medellin. Proceedings of the 10th International Space Syntax Symposium London.

Hillier B. (1996a) Space is the machine. Cambridge University Press.

Hillier B. (1996b) Cities as movement economies. Urban Design International 1(1): 41-60.

Hillier B. (2008) Space and Spatiality: What the built environment needs from social theory. 
Building Research \& Information 36(3): 216-230.

Hillier B and Hanson J. (1984) The social logic of space. Cambridge: Cambridge University Press.

Hillier B, Turner A, Yang T, et al. (2007) Metric and topo-metric properties of urban street networks: some convergences, divergences and new results. 6th International Space Syntax Symposium. Istanbul, 1-21.

Hillier B and Vaughan L. (2007) The city as one thing. Progress in Planning 67(3): 205-230. Holyoak J. (1996) Rational theories for contradictory reality (book review). Architects' Journal 204: 32 .

Jacobs J. (1961) The Death and Life of Great American Cities. New York: Random House. Jiang B and Claramunt C. (2004) Topological analysis of urban street networks. Environment and Planning B 31(1): 151-162.

Law S, Chiaradia A and Schwander C. (2012) Towards a Multi-modal Space Syntax Analysis: A case study of the London street and underground network. 8th International Space Syntax Symposium. Santiago de Chile.

Markus T. (1993) Buildings and Power. London: Routledge.

Marshall S. (2005) Streets \& Patterns. London: Spon Press.

Nagel T. (1998) Reductionism and Antireductionism. The Limits of Reductionism in Biology. Chichester: Wiley, 3-14.

Netto VM. (2016) 'What is space syntax not?' Reflections on space syntax as sociospatial theory. Urban Design International 21(1): 25-40.

Pafka E and Dovey K. (2017) Permeability and Interface Catchment: Measuring and Mapping Walkable Access. Journal of Urbanism 10(2): 150-162.

Porta S, Crucitti P and Latora V. (2006) The network analysis of urban streets: A dual approach. Physica A 369(2): 853-866.

Ratti C. (2004) Space syntax: some inconsistencies. Environment and Planning B 31(4): 487499.

Soja E. (2001) In Different Spaces: Interpreting the spatial organization of societies. 3rd International Space Syntax Symposium. Atlanta, s1.1-s.1.7.

Soria y Puig A. (1999) Cerdá: The Five Bases of the General Theory of Urbanization. Madrid: Electa. 
Steadman P. (2004) "Developments in space syntax". Environment and Planning B: Planning and Design 31(4): 483-486.

Turner A. (2007) "From axial to road-centre lines: a new representation for space syntax and a new model of route choice for transport network analysis." Environment and Planning B: Planning and Design 34(3): 539-555.

Whyte WH. (1980) The social life of small urban spaces. Washington DC: Conservation Foundation.

Elek Pafka is Lecturer in Urban Planning and Urban Design at the University of Melbourne. His research is focused on the relationship between material density, urban form and the intensity of urban life, as well as methods of mapping the 'pulse' of the city. He has a PhD from the University of Melbourne.

Kim Dovey is Professor of Architecture and Urban Design at the University of Melbourne. His research is broadly focused on theories of 'place' and practices of power, covering a broad range of social issues in architecture and urban design. He has a PhD from the University of California, Berkeley.

Gideon DPA Aschwanden is Lecturer of Urban Analytics at the University of Melbourne with a focus on data, learning algorithms and neural networks to evaluate the urban fabric with a focus on health, transportation and economic opportunities. Before the University of Melbourne, he taught and researched at Princeton University on digital fabrication methods and building systems and worked as a researcher at the Future Cities Laboratory in Singapore. He has an MSc in Architecture and a doctoral degree in Science from the ETH Zurich. 